\title{
Recurrent Intussusception in Infants
}

\author{
Ali Ghribi ${ }^{a}$,, Sondes Sahlia ${ }^{a}$ Nada Sghairoun ${ }^{\text {a }}$, Fatma Fitouria ${ }^{a}$, Manef Gasmi ${ }^{a}$, Mourad Hamzaoui ${ }^{a}$
}

\begin{abstract}
Background: The majority of intussusceptions, between 2 months and 2 years, are idiopathic. Indication of surgery in infants with recurrences is still matter for debate. The aim of our study was to review our experience to better define the management of recurrent intussusceptions (RIs).
\end{abstract}

Methods: A retrospective study was conducted involving all patients treated for intussusception at the Department of Pediatric Surgery "A" of Tunis Children's Hospital, over a 5-year period, going from 2008 through 2012. Patients admitted for more than one episode of intussusception were further investigated.

Results: During the 5-year period, 170 infants and children younger than 2 years were diagnosed as having ileocolic intussusception. There were 10 patients with 12 recurrences. Overall recurrence rate was $6 \%$. RI occurred in all cases following successful hydrostatic reduction. Nine infants underwent a second hydrostatic reduction of intussusception. The reduction was successful in all cases. The other infant was taken directly to the operating room because it was too sick to undergo enema. No lead point was found at surgery. The two patients who presented a second recurrence had a successful non-operative reduction. Subsequent laparoscopic exploration was normal in both cases.

Conclusion: Based on our experience and on reviewing the literature, we recommend radiological reduction for more than the third episode of intussusceptions but we must take into consideration the socio-economic level of the parents. Surgery should be reserved for irreducible recurrences and in patients with a suspected pathologic lead point.

Manuscript accepted for publication November 25, 2014

aDepartment of Pediatric Surgery, Children's Hospital, Tunis El Manar University, Place Bab Saadoun 1007, Tunis, Tunisia

bCorresponding Author: Ali Ghribi, Department of Pediatric Surgery, Children's Hospital, Tunis El Manar University, Place Bab Saadoun 1007, Tunis, Tunisia. Email: ali_ghribi_2007@yahoo.fr

doi: http://dx.doi.org/10.14740/ijcp175w
Keywords: Recurrent; Intussusception; Infant; Reduction

\section{Introduction}

Intussusception is a common life-threatening acute abdominal emergency that typically occurs in children between 2 months and 2 years. At this age, the majority of intussusceptions are idiopathic. The recurrence rate following non-operative reduction ranges from $10 \%$ to $15 \%$. Patients with recurrent intussusception (RI) may be subject to repeated enema reductions or surgical intervention. Indication of surgery in infants with recurrences is still matter for debate. We reviewed our experience to better define the management of RI.

\section{Patients and Methods}

A retrospective study was conducted involving all patients treated for intussusception at the Department of Pediatric Surgery of Tunis Children's Hospital, over a 5-year period, going from 2008 through 2012. Information considered included clinical presentation, radiological findings, and results of treatment. Reduction of intussusception by contrast enema was considered successful when there was free and massive reflux of contrast into the small bowel. Patients admitted for more than one episode of intussusception were further investigated. The amount of time between the first episode of intussusception and the recurrent episode was noted.

\section{Results}

During the study period, 170 infants and children younger than 2 years were diagnosed as having ileocolic intussusception. Of this group, 10 patients with 12 recurrences were collected. All these 10 infants had a successful reduction by contrast enema at the first episode of intussusception. Eight children had one recurrence each and two had two RIs. Overall recurrence rate was $6 \%$. There were four boys and six girls $($ sex ratio $=0.66$ ). Age of the 10 infants at the first episode of intussusception ranged from 6 months to 2 years (average, 17.6 months). Sev- 
Table 1. Time to Each Recurrence

\begin{tabular}{lll}
\hline Time interval & Recurrent episodes (n) & Percentage (\%) \\
\hline$<24 \mathrm{~h}$ & 1 & 8 \\
$1-7$ days & 8 & 67 \\
$>1$ month & 3 & 25 \\
\hline
\end{tabular}

en patients were younger than 1 year. Time to each recurrence ranged from 1 day to 8 months (average, 2.9 months). Table 1 shows the time to RI episodes. Seven patients had an RI within 7 days. The earliest recurrence occurred within $24 \mathrm{~h}$, in which the likelihood of incomplete reduction was removed (Fig. 1). Comparison of symptoms occurring at the initial attack and those occurring at recurrent attacks revealed no significant difference (Table 2). Pain and vomiting were the two predominant clinical features. A viral illness was not more prevalent prior to the RI. Average time lag from onset of symptoms until initiation of treatment for the recurrent cases was $20 \mathrm{~h}$, compared with $38 \mathrm{~h}$ for patients with an initial episode. Nine infants underwent a second hydrostatic reduction of intussusception. The reduction was successful in all cases. The other infant was taken directly to the operating room because it was too sick to undergo enema. No lead point was found at surgery. The two patients who presented a second recurrence had a successful non-operative reduction. Subsequent laparoscopic exploration was normal in both cases. No complication related to enema reduction was observed in any RI patient.

\section{Discussion}

$\mathrm{RI}$ is a common sequel after the first intussusception. The reported recurrence rates range from $8 \%$ to $15 \%$. Incidence of RI ranged from $10 \%$ to $15 \%$ after barium or air reduction and from $1 \%$ to $3 \%$ after surgery $[1,2]$. The adhesions resulting from surgery and appendectomy may explain the reduced recurrence rate. In our series of 170 infants, the incidence of $\mathrm{RI}$ after non-operative reduction represented $6 \%$. It was lower than the incidence reported in the literature. Many authors have suggested that the use of pneumatic reduction allows a lower incidence of recurrence in comparison with hydrostatic enema [1]. In our study, seven of 10 patients with RI were younger than 1 year at the first episode and $90 \%$ of the recurrences developed within 6 months of the initial episode. The same findings were found in other series [1,2], which is probably related to the fact that the incidence of intussusception decreases significantly after the toddler period. In our se-

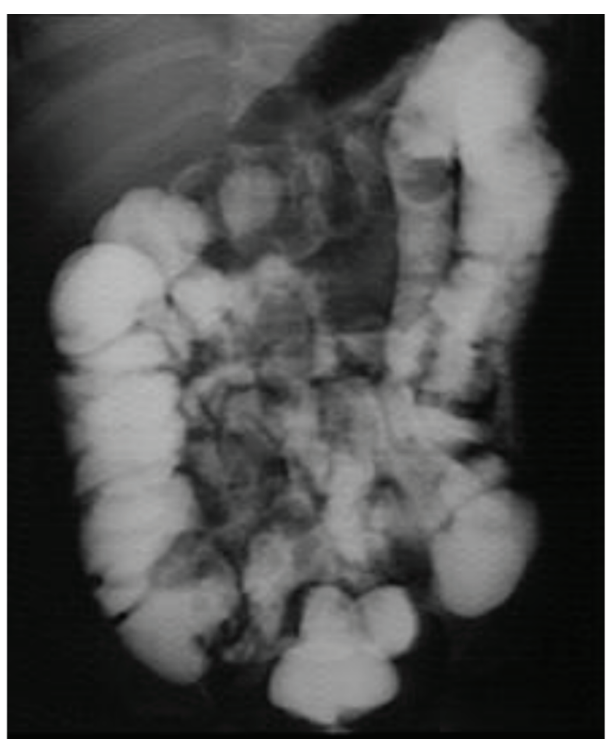

Figure 1. Successful reduction of the initial episode of intussusception by contrast enema for the patient who had presented RI within $24 \mathrm{~h}$.

ries, like in the series of Niramis et al [1], the comparison of symptoms between initial and recurrent attacks revealed no significant difference. Facteau et al [2] found that fewer RI patients presented with signs of advanced intussusception (such as vomiting and blood per rectum). It took much less time on average to make the diagnosis of RI, perhaps because the onset of symptoms alerted the parents to the diagnosis much sooner (Table 3) [1, 2]. The typical symptoms raised the index of suspicion of the parents about a possible recurrence of intussusception. A pathologic lead point (PLP) for the intussusception was identified in $2-12 \%$ of children. A PLP is rarely identified in patients aged less than 2 years. It was found in three infants of 75 RI patients (4\%) in the series of Niramis et al and in nine patients among $114(7.9 \%)$ in the series of Hsu et al [3]. The success rate of enema reduction for RI was close to that for the first episode [4]. Niramis et al [1] found that the success rates after $\mathrm{BE}$ and $\mathrm{AE}$ reduction in recurrent cases were as high as $96 \%$ and $92 \%$, respectively. Reducibility was $95 \%$ for recurrent episodes in the series of Daneman et al [5]. In our study hydrostatic enema was effective in $100 \%$ of cases. The lowest success rate of enema reduction was associated with previous surgery [2]. The number of recurrences that occur before the patient is subject to operative intervention is still open to debate. Hsu et al found, for all ages, that the possibility of RI increased with each recurrence and $100 \%$ of patients with a

Table 2. Clinical Presentations of the Initial and Recurrent Episodes of Intussusception

\begin{tabular}{lll}
\hline Clinical presentation & Initial episodes $(\mathbf{n}=\mathbf{1 0})$ & Recurrent episodes $(\mathbf{n}=\mathbf{1 2})$ \\
\hline Vomiting & 8 & 10 \\
Abdominal pain & 9 & 11 \\
Bloody stools & 5 & 4 \\
Abdominal mass & 3 & 4 \\
History of viral infection & 7 & 6 \\
\hline
\end{tabular}


Table 3. Average Time to Onset of Treatment

\begin{tabular}{lll}
\hline Series & Initial episode & Recurrent episodes \\
\hline Niramis et al [1] & 36 & 17.5 \\
Fecteau et al [2] & 36.5 & 22 \\
Our series & 38 & 20 \\
\hline
\end{tabular}

fourth episode experienced recurrence, so they recommended surgical intervention at the third episode of intussusception [3]. In our department, non-operative reduction was always considered the initial treatment of choice of RI in infants even at the third attack. We often adopted a delayed laparoscopic exploration after reduction of the third episode of intussusception. As for the two patients who underwent surgery, we did not find any lead point. Because of the high reduction rate of recurrences, of the rarity of lead points and of the lack of perforation, we recommend radiological reduction for more than the second RI. But successful reduction does not completely exclude a lead point; for this reason a careful search for PLP is mandatory in RI [5]. Surgery should be reserved for irreducible recurrences, for demonstrated PLP. We think that multiple recurrences do not contraindicate radiological reduction in infants and young children aged $<2$ years but we must also take into consideration the risk of irradiation, the cost of enemas and the socio-economic level of the parents.

\section{Conclusion}

The majority of cases of RI in infants and in children younger than 2 years are idiopathic. We recommend radiological reduc- tion for more than the third episode of intussusception. Surgery should be reserved for irreducible recurrences, in patients with a suspected PLP. We think that several episodes of recurrence at this age do not contraindicate non-operative reduction but we must take into consideration mainly the socio-economic level of the parents.

\section{Conflict of Interest}

The authors declare that they have no conflict of interest.

\section{References}

1. Niramis R, Watanatittan S, Kruatrachue A, Anuntkosol M, Buranakitjaroen V, Rattanasuwan T, Wongtapradit L, et al. Management of recurrent intussusception: nonoperative or operative reduction? J Pediatr Surg. 2010;45(11):2175-2180.

2. Fecteau A, Flageole H, Nguyen LT, Laberge JM, Shaw KS, Guttman FM. Recurrent intussusception: safe use of hydrostatic enema. J Pediatr Surg. 1996;31(6):859-861.

3. Hsu WL, Lee HC, Yeung CY, Chan WT, Jiang CB, Sheu JC, Wang NL, et al. Recurrent Intussusception: when Should Surgical Intervention be performed? Pediatr Neonatol. 2012;53(5):300-303.

4. Ein SH. Recurrent intussusception in children. J Pediatr Surg. 1975;10(5):751-755.

5. Daneman A, Alton DJ, Lobo E, Gravett J, Kim P, Ein SH. Patterns of recurrence of intussusception in children: a 17-year review. Pediatr Radiol. 1998;28(12):913-919. 